\title{
Correspondence
}

\section{TRANSIENT VISUAL LOSS AND OCCULT MIGRAINE}

\section{To the Editor:}

Transient visual loss can be a vexing diagnostic problem. The patient usually presents after the event. If the eye, visual pathways, heart, cranio-cervical vessels, blood viscosity, and coagulation, are all normal, the diagnosis may depend entirely on the history. The importance of oral contraceptive medication and cigarette smoking is not always clear.

Migraine is usually considered when the visual loss was preceded, followed, or accompanied by headache. Sometimes the associated symptoms of migraine (visual loss, paraesthesiae, dysphasia, vertigo) are marked and the headache is minimal or absent. Therefore, an episode of transient monocular or hemianopic visual loss may be the sole manifestation of migraine.

The interval between attacks may be so long that even conscientious patients have forgotten the last episode. Without a history of recurrences the diagnosis is always tenuous. For these reasons it may be worth enquiring about the following facts. They were found to be common and helpful historical features in 3500 patients diagnosed as migraine in the past 30 years. They are, childhood motion sickness, spontaneous prepubertal nosebleeds, chilliness, and a compulsion to eat chocolate.

Motion Sickness: This is probably the commonest childhood symptom associated with migraine in adult life and it may persist throughout life. The patient states that as a child (and occasionally even as an adult) being a passenger in a moving vehicle or on a carnival ride resulted in vertigo, nausea, and vomiting. When the obvious migraineur has no history of early motion sickness he often states that his siblings or parents suffered from it. Chilliness: Most attacks of migraine, painful or otherwise, are preceded or accompanied by a transient, subjective sensation of chilliness. The patient looks cold, pale, and vasoconstricted. Not all patients are aware of this but many subsequently report it after they have been asked about it. Spontaneous Prepubertal Nosebleeds: The association between this complaint and adulthood migraine appears to be greater in males than females. The nosebleeds usually stop after puberty. A Compulsion to Eat Chocolate: This is the least common of the four migraine attributes. Nevertheless, a majority of unequivocal migraineurs report an episodic craving for chocolate in spite of the almost certainty of provoking an attack. Some patients report the craving is strongest when a migraine is about to start or has actually began.

It is not known what proportion of the non-migrainous adult population have these four historical characteristics. Nevertheless, when an otherwise healthy adult has an episode of transient visual loss, even if he denies a past history of headache, if these features are present, the visual loss was probably a migraine manifestation.

R.T. Ross, Winnipeg, Manitoba.

\section{REFINED ROMBERG TEST}

\section{To the Editor}

Romberg's test, described in his "Lehrbuch der Nerven Krankheiten des Menschen $(1846,1853)$ is used as a screening test for diseases of the afferent peripheral nerves or posterior columns. Despite many variations of the test, and differences in opinion over what constitutes a positive test (Rogers 1980) there is agreement that markedly increased swaying or falling with the eyes closed indicates presence of the sign. I have found the addition of horizontal head rotation to the classic Romberg maneuver greatly increases the sensitivity of the test. The revised test is designed to eliminate both vestibular and visual information.

Patients are asked to balance first with eyes open, then closed, and finally with rotation of the head from side to side in a "no" gesture. Four shakes ( 2 to each side) of about 45 degrees are adequate. The sign is judgej as present if the patient sidesteps or opens the eyes to grasp adjacent furniture. Increased swaying is of uncertain significance.

Twenty-eight patients were chosen for testing because of complaints of imbalance. Of 8 patients with clinical evidence of posterior column myelopathy ( 5 inflammatory, 3 spondylitic), 6 did not have the classic Romberg's sign, however, 5 had the sign when head shaking was added. All had mild to moderately diminished vibration sense in the feet and toes, and all but one had diminished joint position sense (JPS), one severely. Of 10 patients with peripheral neuropathy (6Guillain-Barre, 4 diabetic) 7 did not have the classic Romberg's sign but it could be elicited with head shaking in 6 . Two of 3 with normal JPS and slightly impaired vibration sense did not have the classic sign but did have the revised one. Five patients with vestibular dysfunction (brainstem stroke in 2, Meniere's disease, positional vertigo, Ramsay-Hunt syndrome in 1 each) and 5 with cerebellar disease (degeneration in 2, stroke in 2 and phenytoin toxicity in 1) had neither the classic sign nor difficulty with the addition of head shaking. All of the latter two groups had ataxic gait. Thus 11 of 13 patients without the classic Romberg's sign became unbalanced with the addition of head shaking.

In 16 control patients with other types of neurological disease, Romberg's sign and the revised sign were absent. All but one had normal JPS and vibration sense in the feet.

Romberg's original test is not sensitive to loss of JPS or vibration sense since only 5 patients with these deficits had the classic sign. Of interest are 6 of 8 patients with normal or slightly diminished vibration sense and 10 of 12 with normal or slightly diminished JPS who became unbalanced with the addition of head shaking. This suggests that the classic sign and its variants are not directly related to the degree of dysfunction of these sensory systems. It is possible that other larger fiber systems, perhaps spinocerebellar, are more important in maintaining balance when visual and vestibular afferent information are reduced by physical examination maneuvers. The revised sign described here aids in detecting subtle large fiber neuropathy or posterior column myelopathy.

Allan H. Ropper.

Boston, MA.

Rogers J. (1980). Romberg and his test. J Laryngol Otol, 94, 1401-1404.

Romberg M. (1846). Lehrbuch der Nerven Krankheiten des Menschen. A Dunker, Berlin.

Romberg M. (1853). A Manual of the Nervous System. Trans by Sieveking, EGH Sydenham Society London, p 227. 